\title{
Monte Carlo Least-Squares Fitting of the Beam Propagation Factor $M^{2}$
}

\author{
M.B. Alsous*, M. Almezel And M. Alnezami \\ Department of Physics, Atomic Energy Commission, P.O. Box 6091, Damascus, Syria \\ (Received February 19, 2013; in final form July 17, 2013)
}

\begin{abstract}
A new method for $M^{2}$ least-squares fitting using Monte Carlo technique is introduced. The laser beam parameters and the propagation factor $M^{2}$ are calculated according to the ISO 11146 . The initial values of the fitted parameters are analytically calculated and simple relations are given. These relations use three experimental points of the laser beam at different positions. The Monte Carlo fitting algorithm converged rapidly for all the tested beam. The $M^{2}$ value of a diode pumped Nd: $\mathrm{YVO}_{4}$ laser was optimized. The best $M^{2}$ value was 1.08 . This optimization was done by changing the pump spot size at the laser crystal. The best overlapping between the pump spot size and the TEM 00 mode dimension resulted in the best $M^{2}$ value.
\end{abstract}

DOI: $10.12693 /$ APhysPolA.124.673

PACS: 42.60.Jf, 42.55.Xi

\section{Introduction}

The propagation factor $M^{2}$ is an important indicator for evaluating laser beams [1-7]. For many industrial laser applications, a low $M^{2}$ is commonly associated with the ability to focus the beam into a tighter spot, hence, generates finer features. In addition, a low $M^{2}$ results in an increased focus depth when the beam is focused to a particular spot size. This is important because, regardless of the $M^{2}$ of the laser beam, the optics of a system will be chosen to create the focus spot size best-suited to generate the targeted feature size. If high- and low- $M^{2}$ beams are focused to the same optical spot size, then the low- $M^{2}$ beam will have a longer Rayleigh range, hence, a better tolerance for the system defocusing.

Several techniques have been proposed to measure the $M^{2}$, such as the knife-edge method or the variable aperture method [1]. The adopted method by the International Standards Organization (ISO) to determine the laser beam propagation factor $M^{2}$ is based on the second order moments of the power (energy) density distribution as described in ISO 11146 series [5-7]. The proposed beam scanning procedure, with a movement stage, allows beam widths to be sampled as the stage moves through the laser caustic, but, it seems to be an annoying process if many data points are acquired.

Other techniques, with no need to a movement stage, were designed and adopted. They include the measurement of the beam intensity at a fixed plane and behind several rotating lens combinations [8], single image processing using distorted Fresnel zone plates [9], field reconstruction by modal decomposition [10], and digitally simulating the free-space propagation of light using a spatial light modulator [11, 12]. Another approach to measure the $M^{2}$ uses a Shack-Hartmann wave-front sensor, but was shown to yield unpredictable result while multimode beam analysis [13]. According to the ISO stan-

*corresponding author; e-mail: pscientific@aec.org.sy dard, whatever the technique used to measure the beam width, the $M^{2}$ parameter can be determined from a hyperbolic fit.

In this paper, a new fitting method based on the Monte Carlo least-squares technique is proposed to determine the laser beam parameters and to estimate the propagation factor $M^{2}$. Analytical relations are being given for the initial values of the Monte Carlo algorithm. The initial values could be used with any fit algorithm and has accelerated the Monte Carlo least-squares fitting convergence. The developed Monte Carlo method has been applied to calculate the $M^{2}$ factor in a diode pumped solid state laser (DSSL). $M^{2}$ value optimization for a variable pump spot size is discussed.

The reported results in this paper will be useful to laser engineers and scientists working on the design of diode pumped solid state laser devices which operate in single transversal mode.

\section{The Monte Carlo method}

The ISO 11146-1 document states that the beam waist location $z_{0}$, minimum beam width $d_{\sigma 0}$, divergence angle $\theta_{\sigma}$, Rayleigh range $z_{\mathrm{R}}$ and beam propagation ratio $M^{2}$ shall be determined by a hyperbolic fit to different measurements of the beam width $d_{\sigma}$ along the propagation axis $z$. This hyperbolic fit can be expressed in the following way:

$$
d_{\sigma}(z)=f(z, a, b, c)=\sqrt{a+b z+c z^{2}} .
$$

The coefficients $a, b$, and $c$ of the hyperbole shall be determined by least-squares curve-fitting technique. There are several commercial programs for doing this fit, such as Mathematica, Matlab, mathcad or Origin. Other commercial programs are shipped with the beam profilers and beam characterization instruments. Some of these programs would not allow for controlling the convergence parameters of the fitting algorithm (e.g. the step value, the precision or the number of iterations). In this work, a home-written algorithm using the Monte Carlo least-squares fitting technique was developed. 
The implementation of this technique utilizes the random walk method. The corresponding algorithm can then be summarized as follows:

1. A set of initial values for the variables $a, b$, and $c$ are chosen.

2. The error factor $\chi_{0}^{2}$ is calculated using the following formula:

$$
\chi_{0}^{2}=\sum_{i=1}^{i_{\max }}\left[f\left(z_{i}, a, b, c\right)-d_{\sigma}^{\exp }\left(z_{i}\right)\right]^{2},
$$

where $f\left(z_{i}, a, b, c\right)$ is the hyperbolic prediction of the beam width at $z_{i}$ from Eq. (1), and $d_{\sigma}^{\exp }\left(z_{i}\right)$ is the experimentally measured value at $z_{i}$.

3. Each parameter $a, b$, and $c$ is added or subtracted a random amount and subsequently $\chi_{1}^{2}$ is determined. If $\chi_{1}^{2}$ is less than $\chi_{0}^{2}$, the new parameters are adopted as the best-estimated values. Otherwise, the parameters are left unchanged. This process is repeated several times until $\chi^{2}$ cannot get a smaller value than $10^{-4}$.

The random number generator is based on the portable random number generators presented in [14]. The period of the random number sequence is about $2.3 \times 10^{18}$, which is effectively taken to be infinity for this purpose. The initial seeds for the random number generators are generated using the built-in random number generator in Borland $\mathrm{C}++$ Builder [15], which in turn is seeded by the current computer time.

This method converges at a rate $\frac{1}{\sqrt{N}}$, that depends only on the number of tries $N$ regardless of the number of parameters used [14].

\section{Initial values of the fitting process}

The Monte Carlo least-squares fitting algorithm was tested for different experimental laser beams and it converged for all the cases. The convergence speed was essentially dependent on the initial values of the fitted parameters. For some "unrealistic" random initial values, the fitting process took a very long time exceeding $10 \mathrm{~min}$. On the other hand, the time needed for other "realistic" random initial values was few seconds.

To minimize the convergence time, a new method for the determination of the initial values of the fitted parameters $(a, b$, and $c)$ is used. It is based on the analytical solution of the beam hyperbolic equation using three experimental values $\left(z_{i}, d_{\sigma i}\right)_{i=1-3}$. The general hyperbolic propagation law (analog to Eq. (1)) of a laser beam is written as [1-4]:

$$
d_{\sigma}^{2}(z)=d_{\sigma 0}^{2}+M^{4}\left(\frac{4 \lambda}{\pi d_{\sigma 0}}\right)^{2}\left(z-z_{0}\right)^{2},
$$

where $\lambda$ is the laser beam wavelength.

The laser beam parameters can be expressed analytically by solving Eq. (3) and using three experimental values $\left(z_{i}, d_{\sigma i}\right)$ :

$$
z_{0}=\frac{d_{\sigma 3}\left(z_{1}^{2}-z_{2}^{2}\right)+d_{\sigma 1}\left(z_{2}^{2}-z_{3}^{2}\right)+d_{\sigma 2}\left(z_{3}^{2}-z_{1}^{2}\right)}{2\left[d_{\sigma 3}\left(z_{1}-z_{2}\right)+d_{\sigma 1}\left(z_{2}-z_{3}\right)+d_{\sigma 2}\left(z_{3}-z_{1}\right)\right]},
$$

$$
\begin{aligned}
& d_{\sigma 0}=\sqrt{\frac{d_{\sigma 2}^{2}\left(z_{1}-z_{0}\right)^{2}-d_{\sigma 1}^{2}\left(z_{2}-z_{0}\right)^{2}}{\left(z_{1}-z_{0}\right)^{2}-\left(z_{2}-z_{0}\right)^{2}}}, \\
& M^{2}=\sqrt{\frac{d_{\sigma 0}^{2}\left(d_{\sigma 1}^{2}-d_{\sigma 0}^{2}\right)}{\left(\frac{4 \lambda}{\pi}\right)^{2}\left(z_{1}-z_{0}\right)^{2}}} .
\end{aligned}
$$

The divergence angle and the Rayleigh range can be deduced from Eqs. (4) to (6) and are as follows [1-5]:

$$
\begin{aligned}
\theta_{\sigma} & =\frac{4 \lambda}{\pi} \frac{M^{2}}{d_{\sigma 0}}, \\
z_{\mathrm{R}} & =\frac{\pi}{4 \lambda} \frac{d_{\sigma 0}^{2}}{M^{2}} .
\end{aligned}
$$

Hence, all the beam parameters could be calculated for ideal beams using only 3 experimental points. For general beams, it is necessary to perform the least-squares fitting indicated in the ISO document [5] and the above values are used to determine the initial values of the fitted parameters $a, b$, and $c$ :

$$
\begin{aligned}
& c=\frac{d_{\sigma 0}^{2}}{z_{\mathrm{R}}^{2}}, \\
& b=-\frac{2 d_{\sigma 0}^{2} z_{0}}{z_{\mathrm{R}}^{2}}, \\
& a=\frac{d_{\sigma 0}^{2}\left(z_{0}^{2}+z_{\mathrm{R}}^{2}\right)}{z_{\mathrm{R}}^{2}} .
\end{aligned}
$$

This determination of initial values could be used by any fit algorithm or program, and was incorporated in the Monte Carlo least-squares algorithm in a new $\mathrm{C}++$ program. The fitting was tested again for various experimental laser beams and it converged very rapidly for all the tested cases. The convergence time was only a fraction of a second for all the tested beams.

The main advantage of this fit algorithm is the use of the random walk method which can be easily programmed using the parallel programming concept, leading to a very high speed convergence. Therefore, a new 11-thread $\mathrm{C}++$ program has been developed incorporating the Monte Carlo least-squares fit. This new program with 11 parallel threads was tested on a 12-processor workstation. The new convergence time was measured to be the tenth of that of the original one-thread program. This agrees with the fact that the convergence rate of this method $\left(\frac{1}{\sqrt{N}}\right)$ depends only on the number of tries of the algorithm.

For more accurate $M^{2}$ factor value, the user of the program has the choice of excluding the outlying beam widths (some odd points) from the calculation. The exclusion is performed when the distance between the measured and estimated beam diameter exceeds the measurement uncertainty.

\section{Experimental work}

The experimental setup of a $\mathrm{Nd}: \mathrm{YVO}_{4}$ laser is shown schematically in Fig. 1. The pump source was a conductively cooled fiber-coupled diode laser with $200 \mu \mathrm{m}$ core diameter and a numerical aperture of 0.22 . It has a $4.8 \mathrm{~W}$ nominal output power at $806.9 \pm 2 \mathrm{~nm}$. The 
laser crystal was a $2 \mathrm{~mm}$ thick $a$-axis cut $\mathrm{Nd}: \mathrm{YVO}_{4}$ crystal with 1.0 at.\% doping. The input mirror had $1 \mathrm{~m}$ radius of curvature (ROC) with a high transmission at the pump wavelength $(T>98 \%)$ and a high reflectivity $(R>99.8 \%)$ at the laser wavelength $(106 \mu \mathrm{m})$. It was placed $2.5 \mathrm{~cm}$ far from the laser crystal. The output mirror had $1 \mathrm{~m}$ ROC and a reflectivity of $90 \%$ at the laser wavelength. The laser cavity length was $178 \mathrm{~mm}$.

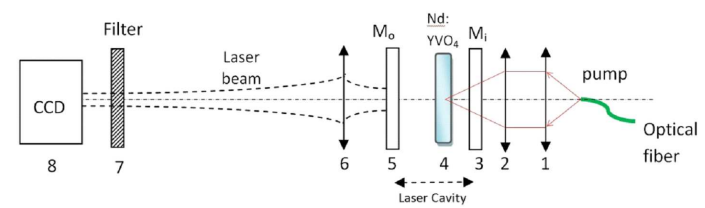

Fig. 1. Experimental setup of $M^{2}$ measurement with a $\mathrm{Nd}: \mathrm{YVO}_{4}$ laser.

The output pump at the fiber end was collimated and focused to a spot diameter of $520 \mu \mathrm{m}$ using two antireflection-coated plano-convex lenses (1) and (2) with $50 \mathrm{~mm}$ and $100 \mathrm{~mm}$ focal length, respectively. Figure 2 shows the pump spot size near the focus of the lens (2) determined by the second order moments of the power.

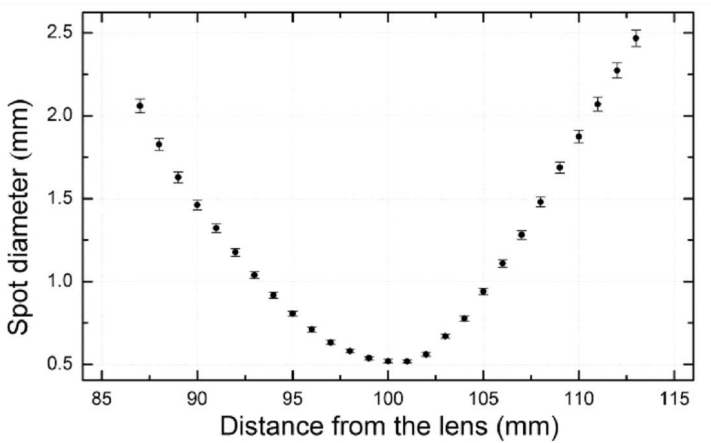

Fig. 2. Pump spot diameters around the focal point.

The Nd: $\mathrm{YVO}_{4}$ laser output was linearly polarized and its maximum $\mathrm{CW}$ power was $1.52 \mathrm{~W}$ at the maximum applied pump power $(4 \mathrm{~W})$, the pump threshold was $1 \mathrm{~W}$, and its slope efficiency was $46 \%$.

A CCD camera was used to measure the second order moment laser beam width. A turning slit beam-profiler was used to ensure the validity of the CCD measurements. The laser beam profile was considered to be of circular symmetry because its ellipticity was found larger than 0.87 . The beam diameter through this work was calculated from

$$
d_{\sigma}(z)=2 \sqrt{2}\left(\sigma_{x}^{2}+\sigma_{y}^{2}\right)^{\frac{1}{2}} .
$$

All the experimental precautions and steps (worming, background, integration area, etc.) mentioned in the ISO document [5] were strictly observed. The measurement of the cross-sectional power density distribution at each location $z$ was repeated five times. A high power Glan cube polarizer was introduced to minimize the laser power reaching the detector. A narrow $10 \mathrm{~nm}$ bandpass filter at the laser wavelength was used to eliminate the residual pump and the surrounding optical noise. A focusing lens $(6)(f=175 \mathrm{~mm})$ was used to create an artificial waist of the beam in order to measure its beam propagation factor. It was verified that none of the optical elements used significantly influenced the relative power density distribution.

The CCD camera was carried on a $50 \mathrm{~cm}$ long optical rail in front of the focusing lens and the measurements of the beam width are taken at more than 10 different $z$ positions along the propagation axis.

The Monte Carlo least-squares fitting was done (Fig. 3) and the best $M^{2}$ value of the $\mathrm{Nd}_{\mathrm{YVO}} \mathrm{YV}_{4}$ laser beam was 1.08. Figure 3 shows that the difference between the initial 3-point hyperbola (dotted line) and the Monte Carlo fit (solid line) is very small.

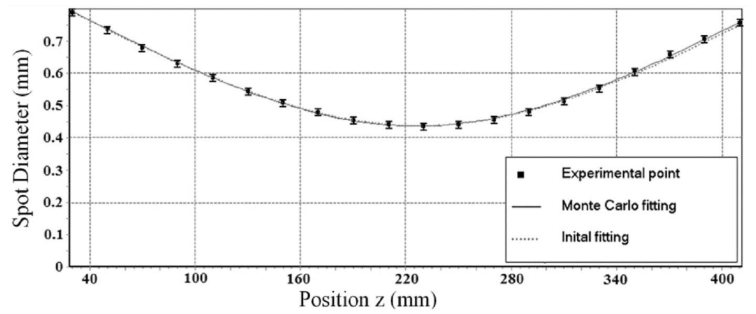

Fig. 3. Experimental data, initial fitting (dotted line) and Monte Carlo fitting (solid line) of the laser beam diameter along the propagation axis.

The $M^{2}$ of the laser beam was calculated for different cavity parameters and pump power values. It was found that the beam factor $M^{2}$ depends strongly on the distance $d$ between the laser crystal and the pump focusing lens (2) as illustrated in Fig. 4 . The best $M^{2}$ value 1.08 was obtained when the $\mathrm{Nd}: \mathrm{YVO}_{4}$ crystal was located $2 \mathrm{~mm}$ before the pump focal point. In addition, for different $d$ values, the best $M^{2}$ did not correspond to the highest output laser power. The output power for the best $M^{2}$ was $1.42 \mathrm{~W}$.

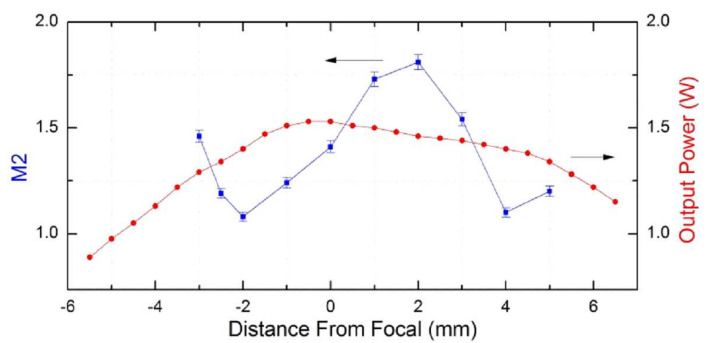

Fig. 4. Monte Carlo estimated $M^{2}$ and output power for different positions of the crystal around the pump focus.

The $A B C D$ method [16-18] was used to calculate the $\mathrm{TEM}_{00}$ mode inside the laser cavity along the two $x$ and $y$ axis. The thermal lens produced inside the laser 
crystal was taken into account as detailed by previous researchers [19-22]. The $\mathrm{TEM}_{00}$ diameter at the laser crystal was found to be about $590 \mu \mathrm{m}$.

Figure 2 illustrated the pump spot diameter dependence on the position of the pump focusing lens (2). The pump spot diameter at the focal point was only $520 \mu \mathrm{m}$, which is smaller than the $\mathrm{TEM}_{00}$ diameter calculated above $(590 \mu \mathrm{m})$. When the laser crystal was displaced $2 \mathrm{~mm}$ further from the pump focal point, the pump size would be enlarged to match the $\mathrm{TEM}_{00}$ dimension. It is concluded that the optimum $M^{2}$ was obtained when the best overlapping between the pump spot size and the $\mathrm{TEM}_{00}$ dimension was realized.

If the laser crystal was displaced $4 \mathrm{~mm}$ closer to the lens, a new good $M^{2}$ value of 1.1 was obtained. This value corresponded to another overlapping between the pump spot size and the $\mathrm{TEM}_{00}$ diameter as indicated in Fig. 2. The first overlapping position, where the laser crystal was placed after the pump focus, resulted in a better $M^{2}$ value and was preferred experimentally.

The pump spot size at the laser crystal is very important for controlling the laser quality. A tight focusing of the pump can increase the local pump density at the crystal resulting in a higher and faster population inversion and a lower laser threshold. The disadvantage of this situation is the high thermal lensing effect that will alter the laser performance and the beam factor at a higher pump rate. On the other hand, a larger pump spot could excite other laser transversal modes resulting in a higher laser gain but with a worst beam factor $M^{2}$ and a higher threshold due to a lower pump concentration.

In general, an aperture is introduced inside the laser cavity to ensure a single transversal mode $\mathrm{TEM}_{00}$ oscillation [16, 23]. This aperture will drastically reduce the laser output. Instead, the size matching between the pump spot and the $\mathrm{TEM}_{00}$ mode would allow for a total use of the pump resulting in higher laser efficiency and only $6.5 \%$ loss of the total output power.

\section{Conclusions}

The Monte Carlo least-squares fitting technique was used to determine the laser beam parameters and the propagation factor $M^{2}$ according to the ISO 11146 documents. Simple analytical relations were introduced to calculate the fit initial values. These relations are based on three experimental spot diameters at different positions. This allowed for a better and faster convergence of the fitting algorithm.

The $M^{2}$ value was calculated for a diode end-pumped $\mathrm{Nd}: \mathrm{YVO}_{4}$ solid state laser. The $M^{2}$ value was optimized by changing the pump spot size at the laser crystal and the best $M^{2}$ found was 1.08 . This value corresponds to the best overlapping between the pump spot size and the $\mathrm{TEM}_{00}$ mode diameter at the laser crystal.

\section{Acknowledgments}

The authors would like to thank Prof. I. Othman, the Director General of the AECS, for his support and encouragement. Thanks are also due to Dr. M.D. Zidan from Laser Lab. and to Dr. H. Jouhara for the valuable discussions.

\section{References}

[1] A.E. Siegman, M.W. Sasnett, T.F. Johnston, IEEE J. Quant. Electron. 27, 1098 (1991).

[2] R. Borghi, M. Santarsiero, Opt. Lett. 22, 262 (1997).

[3] A.E. Siegman, "How to (Maybe) Measure Laser Beam Quality", REOL, April 2004, presented at OSA Annual Meeting, 1998.

[4] A.E. Siegman, S.W. Townsend, IEEE J. Quant. Electron. 29, 1212 (1993).

[5] ISO 11146-1:2005 "Lasers and laser-related equipment - Test methods for laser beam parameters - Beam widths divergence angle and beam propagation factor".

[6] ISO 11146-2:2005 "Lasers and laser related equipment - Test methods for laser beam widths divergence angles and beam propagation ratios General Astigmatic beams".

[7] ISO/TR 11146-3:2004 "Lasers and laser related equipment - Test methods for laser beam widths divergence angles and beam propagation ratios. Intrinsic and geometrical laser beam classification propagation and details of test methods".

[8] G. Nemes, A.E. Siegman, J. Opt. Soc. Am. A 11, 2257 (1994)

[9] R. Cortés, R. Villagómez, V. Coello, R. López, Revista Mexicana De Fúsica 54, 279 (2008).

[10] O.A. Schmidt, C. Schulze, D. Flamm, R. Brüning, T. Kaiser, S. Schröter, M. Duparré, Opt. Expr. 19, 6741 (2011).

[11] C. Schulze, D. Flamm, M. Duparré, A. Forbes, Opt. Lett. 37, 4687 (2012).

[12] J. Pérez-Vizcaíno, O. Mendoza-Yero, R. MartínezCuenca, L. Martínez-León, E. Tajahuerce, J. Lancis, J. Displ. Techn. 8, 539 (2012).

[13] J.V. Sheldakova, A.V. Kudryashov, V.Y. Zavalova, T.Y. Cherezova, Proc. SPIE 6452, 645207 (2007).

[14] W.H. Press, S.A. Teukolsky, W.T. Vetterling, $N u$ merical Recipes in $\mathrm{C}++$, Cambridge University Press, New York 2007.

[15] C. Calvert, Borland $C++$ Builder Unleashed, Borland Press, Indianapolis 1998.

[16] N. Hodgson, H. Weber, Optical Resonators, Springer, New York 1996.

[17] A.E. Siegman, Lasers, Oxford Univ. Press, California 1986.

[18] P. Das, Lasers and Optical Engineering, Springer, New York 1990.

[19] Z. Xiong, G. Li, N. Moore, W.L. Huang, G.C. Lim, IEEE J. Quant. Electron. 39, 979 (2003).

[20] B. Neuenschwander, R. Weber, H.P. Weber, IEEE J Quant. Electron. 31, 1082 (1995).

[21] J. Zheng, S. Zhao, L. Chen, Opt. Laser Technol. 34 , 439 (2002).

[22] P.K. Mukhopadhyay, K. Ranganathan, J. George, S.K. Sharma, T.P.S. Nathan, Opt. Laser Technol. 34, 253 (2002).

[23] W. Koechner, Solid-State Laser Engineering, Springer, New York 2006 